\title{
Percutaneous interventions in stenotic valvular heart disease complicating pregnancy
}

\author{
Its risks are much less than those of surgery or continuing pregnancy on medical therapy
}

Heart disease complicating pregnancy is a common and serious clinical condition. Cardiac disease does not preclude pregnancy but increases the risk to both the mother and the foetus, despite state-of-the-art obstetric and cardiac care. The incidence of maternal heart disease in Sri Lanka is about 1.5 per hundred deliveries. About $70 \%$ are of rheumatic origin [1]. It causes chronic valvular lesions commonly affecting the mitral and aortic valves. Since the natural history of rheumatic mitral stenosis typically has a 20-25 year latent period symptoms often appear first during pregnancy [2].

Women with stenotic valvular heart disease have a significantly higher incidence of congestive heart failure (38\% vs $0 \%, \mathrm{p}<0.00001)$, arrhythmias $(15 \%$ vs $0 \%$, $\mathrm{p}<0.002)$, initiation or increase of cardiac medications (41\% vs $2 \%, \mathrm{p}<0.0001$ ), and hospitalisations (35\% vs $2 \%, \mathrm{p}<0.0001)$. Moreover, it has an effect on foetal outcome, resulting in increased preterm delivery $(23 \%$ vs $6 \%, \mathrm{p}<0.03)$, intrauterine growth retardation $(21 \%$ vs $0 \%, \mathrm{p}<0.0001$ ), and a reduced birth weight [3].

During pregnancy the haemodynamic changes result in a steady increase in cardiac output until the 32nd week, at which time cardiac output plateaus at $30 \%$ to $50 \%$ above the pre-pregnancy level. This haemodynamic stress, along with other factors such as anaemia and atrial fibrillation (AF), may precipitate acute pulmonary oedema with unacceptable maternal and foetal mortality.

During labour uterine contractions $300-500 \mathrm{~mL}$ of blood are released into the circulation, causing an additional increase in blood volume. This, together with increased blood pressure and heart rate during labour further increases cardiac output. At the time of delivery it increases to as much as $180 \%$ of the pre-pregnancy level. Because of the haemodynamic changes that occur during pregnancy obstructive cardiac lesions are generally poorly tolerated. However, regurgitant lesions are tolerated relatively well because of the decrease in systemic vascular resistance.

Hence critical stenotic valvular lesions should be treated before delivery, ideally during the second or third trimester, to improve pregnancy outcome. Vaginal delivery with a facilitated second stage (forceps delivery or vacuum extraction) is indicated for women with cardiac disease unless there are obstetric reasons to recommend caesarean section. Optimal anaesthesia and analgesia and administration of prophylactic treatment for endocarditis should be considered before delivery. Haemodynamic monitoring is recommended for high-risk patients with severe or symptomatic maternal cardiac disease.

Maternal prognosis during pregnancy is related to functional class. Maternal mortality for New York Heart Association (NYHA) class I and II is less than 1\%. With
NYHA class III and IV maternal mortality increases up to $7 \%$ [4-6]. It can go as high as $17 \%$ in patients with AF $[5,6]$. The highest risk occurs during labour and delivery. Foetal mortality is also related to maternal functional class and the rate is $30 \%$ for women with NYHA class IV.

The management of pregnant women in NYHA class I or II should include limiting strenuous exercise, having adequate sleep and rest, maintaining a low salt diet, avoiding anaemia (maintain haemoglobin > $11 \mathrm{~g} / \mathrm{dL}$ ), having frequent prenatal examinations (both obstetric and cardiovascular), and monitoring for arrhythmias. In more symptomatic women (NYHA class III or IV) pregnancy should be avoided or interrupted. Should pregnancy occur bed rest is often required, particularly during the latter part of the pregnancy, and close cardiac and obstetrical monitoring is essential.

\section{Mitral stenosis}

Mitral stenosis is the commonest cardiac lesion seen during pregnancy. Patients with symptomatic mitral stenosis are usually managed with medications such as digoxin, frusemide, KCI and beta blockers. Patients with moderate to tight mitral stenosis with inadequate response to pharmacotherapy should be referred for surgical or percutaneous intervention (ballon valvuloplasty).

Over the past few decades closed mitral valvotomy has been the mainstay of therapy for tight mitral stenosis. Patients have benefited significantly from this procedure. Numerous studies over the past three decades have documented the efficacy and safety of this surgical procedure though it carries significant foetal morbidity and mortality $[7,8]$. Open heart surgery during pregnancy has a $33 \%$ risk of foetal mortality. With closed mitral commisurotomy the risk of foetal morbidity and mortality is much lower, although a 5-15\% risk of foetal death still exists [9]. Open mitral valvotomy is also associated with a high maternal mortality $[9,10]$.

Since its introduction in 1984 percutaneous transvenous mitral commissurotomy (PTMC) numerous studies have established the efficacy and the safety of this procedure [10-13]. Over the last few years PTMC has been done in several centres worldwide with excellent short term results in patients with mitral stenosis during pregnancy. For patients in the second and third trimester of pregnancy PTMC would be expected to achieve haemodynamic and symptomatic improvement with minimal risk to the mother and foetus. Though there are several techniques available the Inoue technique has been the most popular in view of its low complication and high success rate. 
Long term follow up studies have shown good outcome of pregnancies, with no significant problems in the foetus. Recent data on Sri Lankan patients with symptomatic mitral stenosis (pregnant and non-pregnant) showed excellent results (success rate $>96 \%$ ) and very low complication rates with PTMC $[14,15]$.

Mild to moderate aortic regurgitation or mild to moderate mitral regurgitation are not contraindications for PTMC if valve morphology is suitable for intervention. Patients with severe calcific mitral stenosis, left atrial thrombus and more than moderate mitral regurgitation are not considered for this procedure. But patients with a large left atrium, aneurysmal interatrial septum and even severe pulmonary hypertension could be subjected to balloon mitral valvuloplasty.

Ballon mitral valvuloplasty is carried out under fluoroscopic screening in the angio-cardiographic laboratory, with local anaesthesia. All patients are adequately covered by a lead shield to protect the uterus and minimise radiation hazard to the foetus.

PTMC has many advantages over surgery. Following PTMC the hospital stay is about $24 \mathrm{~h}$ compared to 1 or 2 two weeks in cases of closed mitral valvotomy. PTMC patients are mobilised on the same day and discharged the following day. As PTMC does not involve general anaesthesia or surgery it avoids the risks of these procedures. Furthermore, one can demonstrate the haemodynamic improvement immediately after the procedure. The short time taken for PTMC is also an advantage.

Radiation exposure during fluoroscopy may pose a potential hazard to the unborn child. The foetus receives most of the radiation dose from scatter, and this has been estimated as approximately $0.2 \mathrm{rad}$ during balloon mitral commissurotomy [16]. Therapeutic abortion is recommended when the foetus is exposed to $10 \mathrm{rad}$ or more. Since foetal abnormalities are more likely to occur with radiation exposure before 20 weeks of gestation $[17,18]$, PTMC is recommended routinely after 20 weeks of gestation. A recent study has shown that over a follow up of more than 5 years, all children born to women who had undergone PTMC during pregnancy showed normal growth and development without any clinical abnormalities [19]. Risks to the foetus associated with exposure to radiation may be avoided by using transoesophageal echocardiography to guide the procedure instead of fluoroscopy.

\section{Aortic stenosis}

Symptomatic aortic valve disease is less common than mitral valve disease in pregnant women, and rheumatic heart disease is the most common cause in developing countries. The pressure gradient across the aortic valve is responsible for the haemodynamic changes in aortic stenosis. The increase in left ventricular systolic pressure needed to maintain sufficient pressure in arterial circulation leads to increased stress on the ventricular wall with compensatory left ventricular hypertrophy. Symptoms develop when compensatory changes in the left ventricle are inadequate to meet the demands imposed by the need for increased cardiac output late in pregnancy. This usually occurs with moderate to severe aortic stenosis.

The severity of the condition and its symptoms largely determine management of aortic stenosis. Most asymptomatic patients and those who have mild to moderate stenosis can be managed with medical therapy. Patients who are symptomatic or who have a peak outflow gradient of more than $50 \mathrm{mmHg}$ are advised to delay conception until after surgical correction. Termination of pregnancy should be strongly considered if the patient is symptomatic before the end of the first trimester.

Percutaneous balloon valvuloplasty and aortic valve replacement are the available options for management. However, several studies of balloon valvuloplasty for severe aortic stenosis during pregnancy suggest favourable outcomes for both mother and foetus. Balloon valvuloplasty is not the preferred treatment in patients with calcified aortic valves or in the presence of significant aortic regurgitation. Avoidance of severe vasodilation and maintenance of an adequate fluid balance are vital to ensure that cardiac output is not compromised. Low epidural anaesthesia may be used to minimise vasodilatory effects.

\section{Conclusion}

The risks of balloon valvuloplasty are much less than the risks of either surgery or continuing the pregnancy on medical therapy in women with symptomatic valvular lesions without contraindications. They should be treated with percutaneous interventions. But our primary aim should be directed at early detection, assessment and appropriate measures before conception.

\section{References}

1. Kaluarachchi A, Seneviratne H.R. Heart disease complicating pregnancy: evaluation of disease pattern in Sri Lanka, Journal of Obstetrics and Gynaecology 1995; 15: 9-14.

2. Prasad AK, Ventura HO. Valvular heart disease and pregnancy. Postgraduate Medicine 2001; 110: 33-7.

3. Afshan Hameed, Ilyas S Karaalp, Padmini P Tummala, et al. Effect of valvular heart disease on maternal and foetal outcome of pregnancy. Journal of American College of Cardiology 2001, 37: 893-9.

4. Metcalfe J, Ueland K. Maternal cardiovascular adjustments to pregnancy. Progress in Cardiovascular Diseases 1974; 16: 363-74.

5. Szekely P, Turner R, Snaith L. Pregnancy and changing pattern of rheumatic heart disease. British Heart Journal 1973; 35: 1293-1303.

6. Perloff JK. Pregnancy and cardiovascular disease. In: Braunwald E, ed. Heart Disease. A Textbook of Cardiovascular Medicine. 5th ed. Philadelphia: WB Saunders, 1997: 1843-64. 
7. Knapp RC, Arditi LI. Closed mitral valvotomy in pregnancy. Clinical Obstetrics and Gynaecology 1968, 11: $978-91$.

8. Vosloo S, Reichart B. The feasibility of closed mitral valvotomy in pregnancy. The Journal of Thoracic and Cardiovascular Surgery 1987, 93: 675-9.

9. Bernal JM, Miralles PJ. Cardiac surgery with cardiopulomary bypass during pregnancy. Obstetrical and Gynaecological Survey 1986; 41: 1-6.

10. Smith R, Brender D, Mc Credie M. Percutaneous transluminal mitral valve dilatation in pregnancy. British Heart Journal 1989; 61: 551-3.

11. Ben Farhat M, Maatouk F, Betbuot F, Ayari M, Brahim $\mathrm{H}$, et al. Percutaneous balloon mitral valvuloplasty in 8 pregnant women with tight mitral stenosis. European Heart Journal 1992; 13: 1658-64.

12. Ben Farhat M, Gamra H, Betbuot F, Maatouk F, Jarrar $\mathrm{M}$, et al. Precutaneous balloon commissurotomy during pregnancy. Heart 1997; 77: 564-7.

13. Mishra S, Narayang R, Sharma M, Chopra A. Percutaneous transseptal mitral commisuromy in pregnant women with critical mitral stenosis. Indian Heart Journal 2001; 53: 192-6.

14. Senaratne V. Balloon mitral valvuloplasty-a new approach in the management of mitral stenosis. Journal of the Ceylon College Physicians 2000; 33: 100-9.

15. Senaratne V. Immediate Results of Balloon Mitral Valvuloplasty with Inoue Balloon: a Sri Lankan Experience. Scientific Abstracts. 13th ASEAN Congress of Cardiology in Singapore, 2000.

16. Safian R, Berman A, Sachs B, Diver DJ, Com PC, et al. Percutaneous balloon mitral valvuloplasty in a pregnant women with mitral stenosis. Catheterization and Cardiovascular Diagnosis 1988; 15: 103-8.

17. Brent RL. The effect of embryonic and fetal exposure to $\mathrm{x}$-ray microwaves and ultrasound: counselling the pregnant and nonpregnant patient about these risks. Seminars in Oncology 1989; 16: 347-68.

18. Gray JE. The radiation hazard: let's put it in perspective. Mayo Clinic Proceedings 1979, 54: 809-13.

19. Mangione JA, Lourenco RM, dos Santos ES, Shigueyuki A, Mauro MF, et al. Long term follow up of pregnant women after percutaneous mitral valvuloplasty. Catheterization and Cardiovascular Interventions 2000; 50: 413-7.

Vajira Senaratne, Resident Cardiologist, Institute of Cardiology, National Hospital, Colombo. Tel: +94 0777353146, e-mail: vajiras@telenett.net. (Competing interests: none declared). 